\title{
Study on Carving Art of DangShi Manor in Suide County, Shaanxi, China
}

\author{
Yanjun Li \\ College of Art and Design, Xi'an University of Technology \\ No.58 Yanxiang Road, Xi'an 710054, Shaanxi, China \\ Tel: 86-29-8206-6390 E-mail: liyanjun2227478@yahoo.com.cn
}

\author{
Xiaosheng Sun \\ College of Art and Design, Xi'an University of Technology \\ No.58 Yanxiang Road, Xi'an 710054, Shaanxi, China
}

Tel: 86-29-8206-6427Ｅ-mail: sunxiaosheng148@163.com

\author{
Received: November 28, $2011 \quad$ Accepted: December 28, $2011 \quad$ Published: January 1, 2012 \\ doi:10.5539/ach.v4n1p48 \\ URL: http://dx.doi.org/10.5539/ach.v4n1p48
}

\begin{abstract}
The DangShi manor is located in Hejiashi Village, Baijiajian Town approximately 20 kilometers from the southeast part of Suide County, Shaanxi Province, the unique architecture in Qing Dynasty that has been preserved almost intact in Suide County, a great cultural county, and is an officially protected site in Shaanxi Province. By means of field survey, mapping and taking photos and recording in Dangshi manor, this article acquires the abundant first-hand data about carving art of the manor. With the methods of comparison and induction from the holistic perspective and detailed analysis in the local perspective, this article makes a meticulous analysis and study on the style, content, connotation, moulding and technique of carving art of Dangshi manor and discusses characteristics and protection and inheritance value of carving art of Dangshi manor in a systematic way.
\end{abstract}

Keywords: Suide County, Dangshi manor, Carving art, Study

Shaanxi is a province in the northwest part of China and can be divided into three major areas from the north to the west, respectively, Shaanbei (northern Shaanxi), Guanzhong (the central Shaanxi) and Shaannan (southern Shaanxi). Shaanbei is located in the central area of the loess plateau of China, in which the loess soil texture is widely distributed and continuous, which, together with the strong uprightness, offers important congenital conditions for construction of cave dwellings. Meanwhile, under the influence of the traditional economy and culture for thousands of years, construction of cave dwellings has been optimized and evolved continuously and gradually folk cave dwelling construction with deep traditional context is formed. Dangshi manor, which is located in the southeast part of Suide County in Shaanbei, is a castle folk cave dwelling architectural complex with strong regional characteristics (See Figure 1). It has extremely high research value and realistic meaning in terms of architecture, aesthetics and resident culture, etc., in the multiple aspects of its scientific and skillful architectural composition, spatial organization and rich decoration elements, etc. The stone carving, tile carving and wood carving that are decorated in the manor are the major engraving decoration patterns of the manor. Considering the manifestation content, distribution pattern, connotation and engraving technique of the carvings, the Chinese traditional architectural and cultural quintessence and belief are embodied. The abundant and delicate engraving and decorating elements are all god-given folk rare arts. Thus, this article is going to make an analysis from the "three carvings" (stone carving, tile carving and wood carving) in Dangshi manor.

\section{Stone Carving}

Suide is a well-known stone carving county in Shaanbei and even in the whole country. Owing to its particular geographical position and congenital advantages, Suide has created abundant mountainous and stone resources 
as well as resources of human talents (Baidu Wikipedia). Furthermore, with its historical and cultural sediment for thousands of years, the stone carving art in Suide enjoys tremendous popularity in the world owing to delicate engraving, profound implication and a great variety, etc. As one of the major engraving patterns in the manor, the art of stone carving naturally reflects everywhere the artistic features of local excellent traditional carving under the great cultural background as a county of stone carving art. The stone carving mainly has such structures of bearing stone, milling utensil, plinth, screen wall, memorial archway and so on as its carriers, with various engraving skills of relief, open work carving, circular engraving and so on, colorful manifestation content and adept skills. All the above reveals the excellent skills and rich imagination of local stonemasons. Stone carving in Suide is a god-given artistic treasure in the engraving arts of the Chinese nation.

Most stone carving structures that are preserved intact in the manor are bearing stone, plinth and drum-shaped bearing stone which are various in the theme of engraving and employ a variety of materials, such as, dermatoglyphic patterns of animals and plants. Among all the structures, engraving of bearing stone is most outstanding. Commonly referred to as gate pier, gate seat, gate platform and gate stone, bearing stone is a functional structure that is installed on the both sides inside and outside the threshold to fix the spindle of the door leaf. As it is engraved in the shape of a pillow or a box, it is thus termed as bearing stone (Wu Wei, 2006). Bearing stone on the two sides of NO. 3 courtyard and NO. 5 countryard in the main residential area of Dangshi manor are quite typical (See Figure 2). The bearing stone is engraved with an entire bluestone and is mainly divided into three parts from bottom to the top. The top part is usually engraved with two prostrate lions, with an appearance either silly and childlike, or rough and mighty. The middle part is engraved with the deep relief skill on the surface, with the patterns of "Si Ji Ping An" and "Ping An Ru Yi", which stand for people's blessing for a beautiful life of happiness in the four seasons and one in which everything goes well. The middle part is also engraved with the pattern of "Bo Gu" and "Si Yi" that are filled with full-bodied primitive simplicity and elegance. The bottom part is engraved with such auspicious patterns as "Feng Chuan Mu Dan" and "Yuan Yang $\mathrm{Xi} \mathrm{He",} \mathrm{with} \mathrm{the} \mathrm{implication} \mathrm{of} \mathrm{happiness} \mathrm{and} \mathrm{brightness,} \mathrm{harmony} \mathrm{in} \mathrm{the} \mathrm{couples} \mathrm{and} \mathrm{deep} \mathrm{love} \mathrm{to} \mathrm{each} \mathrm{other.} \mathrm{In}$ addition, the Chinese dragon that is generally termed as "benevolent beast" in the traditional Chinese engraving art, the bat and the sika deer that symbolize happiness are also engraved on the stones. In addition to the bearing stone, the plinth and milling utensil are also engraved with some auspicious patterns with the skill of shallow relief. According to introduction by Dang Jingde, descendant of Dangshi manor, a grand and delicately engraved stone stele ever stood erect at the major entrance of the manor with complex and beautiful engraving skills which was used to record honor or disgrace and ups and downs of the clan. Yet, it is a great pity that the stone stele was destroyed during the "Great Cultural Revolution". It can be seen that, whether in terms of engraving skills or in terms of engraving content, the stone decoration art of Dangshi manor can be said to be a competitive art product in the traditional folk building of Shaanbei.

\section{Tile Carving}

Tile carving is also called "Yinghuahuo", in which engraving is made with instrument and with black brick as the material and in which such images as person, animal, flower and bird are regarded as the major content of constructing decoration ( $\mathrm{Li}$ Yanjun, 2011). The manifestation content is mainly character allusions and auspicious patterns that people are delighted to hear and see, which vividly expresses people's blessing for a beautiful life. The tile carving art of Dangshi manor employs a lot of engraving technique and skills and a variety of colorful expression themes, which leaves a lot of exquisite tile carving works for the later generation and is a precious artistic treasure in the traditional Chinese culture.

The tile carving art of Dangshi manor is mainly reflected in such architectural elements as screen wall, ridge of a house, Chitou, baldachine and so on, with various and abundant engraving materials of engraving content and themes, such as, character story, fish, insect, flower and plant, auspicious bird and beast, auspicious appearance pattern. All these patterns reflect blessing of people from the Dangshi family for a happy life and their hope for prolong life and their beautiful wish for wealth and rank. In terms of engraving technique, tile carving is similar to stone carving in that both of the two carvings employ relief, flat carving, open work carving and so on which are some common engraving skills and which are exquisite, skillful. There is no lack of reality in exaggeration and there is no loss of ingenuity and smoothness in roughness, which manifests the engraving style with features of Shaanbei in an intact way.

Among the multiple tile carving works within the Dangshi manor, the most characteristic one is the tile carving art of screen wall. Screen wall is a sort of architectural form with special features that is generated under the influence of the geomantic omen in the traditional Chinese culture. located inside or outside the courtyard as a short wall of a protective screen, the screen wall is also called "entrance screen". In addition to its inherent functions of keeping out of the wind and shielding the vision, etc., the screen wall more reflects the customs and 
beliefs, supernatural exorcism, enjoying life of ease and comfort, reproduction and propagation, caution and indoctrination, etc., reflecting a harmonious relationship between human and the nature, namely, "theory that man is an integral part of the nature" (Zhang Yuncun, 2009). There are three screen walls that are kept intact so far within Dangshi manor (See Figure 3). All of the three screen walls are different in distribution pattern. Two of the three screen walls are located right opposite the gate of NO. 8 courtyard and NO. 18 Courtyard in the major residential area and the third screen wall is located beside the gate of NO. 3 courtyard. Different distribution patterns not only guarantee quietness and privacy within the courtyard, but also helps exorcise evil spirits in terms of the geomantic omen. The engraving content and themes on the screen wall of Dangshi manor are also extensive. On the top of the screen wall is laid semicircle-shaped tile, the central part is laid with a ridge. On the both ends of the central ridge is a roof charm, below the cornice is a rafter and a bracket system, with the same structure and decoration as the roof. There are also some dermatoglyphic patterns and zigzag patterns. Between the bracket system and the bracket system are often engraved with such character patterns as "God of Wealth" and "Child Born out of Lotus", etc., which mostly employ the engraving skills of relief and circular engraving. On the main body of the screen wall, the skill of relief is employed in decoration of the tile body in the central screen wall heart, engraved with such patterns of "Feng Drama Peony" or Chinese auspicious characters of "Fu" and "Shou". Or the screen wall center is engraved with turtleback pattern as the background and the center is engraved with the three auspicious Chinese characters "Fu, Lu Shou, with the implications of longevity and happiness, etc.. The four corners of the top, bottom, left and right of the screen wall center are engraved with auspicious patterns of flowers and plants, which makes the entire screen wall look more elegant.

In addition to tile carving art in such architectural elements as screen wall, tile carving in Chitou, ridge, eaves tile and the triangle-shaped edge is also an important component in tile carving art of Dangshi manor (See Figure 4). Most of the engraving and decoration content is flowers and plants, fish and insect, immortal characters and auspicious bird and beasts. The wood carving art in Chitou is the most prominent and the engraving technique is mainly relief and circular engraving, with a variety of themes, such as, "Fu, Lu, Shou, Xi", "Xi Shang Mei Shao", "Yuan Yang Xi He", "Lian Sheng Gui Zi" and "Er Long Peng Shou", etc. The engraving technique is delicate and skillful, with profound implications. The four corners of the base of Chitou are also engraved with the pattern of an elegant, conveying an auspicious meaning. In addition, the ridge and the eaves tile are respectively engraved with a dragon-head ridge ornament with the implication of water proof and disaster prevention, a tree peony which symbolizes wealth and rank and bat which symbolizes happiness, all of which fully reveals the traditional profound culture deposits of Northern China.

\section{Wood Carving}

Wood carving is an important decoration technique in architectural elements, which is reflected everywhere in gate cover, window sash, wood partitions, board attached to a wall and furniture, etc. Wood carving technique is centered by relief technique, which is also combined with open work carving and three-dimensional thorough carving. Mostly, the clear water technique is used, and there is also color decoration technique (Yue Shijun, 2010). Wood carving art has had a long history of development. Especially, the wood carving art in Ming Dynasty and Qing Dynasty can be said to have attained the peak in terms of engraving technique, content theme and quantity, etc. Dangshi manor was set up in Ming Dynasty and Qing Dynasty. As one of the representatives of residential cave buildings in Shaanbei, the wood carving and decoration art is deeply affected by the rich and traditional culture of Northern China, with succinct, bold and unrestrained, skillful and delicate features. Wood carving is also widely distributed in terms of architectural ornament, which employs a lot of engraving skills to present all sort of themes and stories in a lifelike way. Besides, the wood carving is well arranged, full and substantial and clear-cut in texture. Since it has had a long history and it was seriously damaged during the Great Cultural Revolution, it is a pity that some parts of wood carving in the Dangshi manor have been destructed.

Wood carving art of Dangshi manor is mainly concentrated in such architectural elements as sparrow brace, forehead wood, adjacent of windows, doors and windows and so on. A variety of engraving techniques are adopted according to different positions and the engraving techniques of bas-relief, deep relief, open work carving, circular engraving and hollowing carving are alternated and combined, which greatly enriches formation of the pattern of wood carving art in Dangshi manor. Furthermore, the engraving content with various and profound implications makes the elegant cultural flavor of the manor most incisive.

Gate tower is a place where decoration of folk dwellings in Shaanbei area is special and it is a sign of entrance for each household. Moulding of the gate tower and precision in the work of engraving reveals the decoration quality of the entire house and the identity and social status as well as family background of the head of the household. Therefore, gate tower is an important position which the heads of households take pains to depict ( $\mathrm{Li}$ Meng, 2006). Naturally, the wooden architectural element on the lintel of a door becomes an important position 
of engraving. The wooden engraving and decoration structure of sparrow brace and forehead wood in Dangshi manor is skilful, various in pattern and also versatile in patterns of engraving. Some employ the technique of flat carving, some employ the technique of hollowing carving to avoid tediousness in vision and use moulding of animals and plants, such as, crane, Chinese dragon, magpie, mandarin duck, legendary phoenix bird, lotus, tree peony and lotus flower, etc. There are some which also use simple fretwork and bat pattern, etc., which is matched with beautiful colored drawing, with the content of natural scenery or ancient character legend and story, etc. In addition, the position of gate plaque is engraved with such Chinese characters as "Fu", "Shou" or "Gengdu" and "Wukui" etc., expressing people's yearning for happiness and longevity and the eagerness to display the prominent status of the family. Collocation of multiple engraving patterns greatly riches artistic formation of the lintel of a door and leaves a profound impression on people.

In addition to the gate tower, the most typical door and window pattern in Shaanbei area is arch door connected with window, which is also the most special part in decoration of folk houses in Shaanbei. According to primary and secondary caves, the wood carving art of the caves also exhibit different complexities and dermatoglyphic patterns. NO. 3 courtyard that has been kept relatively intact in the major residential area of Dangshi manor is a traditional layout form of "three bright rooms, two dark rooms and two wing-rooms" that is the most typical in cave folk dwellings in Shaanbei. Decoration of doors and windows of the major cave is the most complicated and has the most special engraving, with delicate artistic work. Doors and windows are engraved with such patterns as single corner pattern, lantern frame, auspicious knot, Koutian lattice. Danzai lattice and turtle back pattern, etc. The position of door lintel is also engraved in the hollowing carving form with the Chinese character as "Shou(寿)", butterfly, pumpkin and Aquarius, which are patterns symbolizing longevity and auspiciousness (See Figure 5). By contrast, doors and windows of the secondary caves are relatively simply decorated, which are usually engraved with patterns of diamond lattice or square lattice. And almost all doors and windows are based on the theme of symmetry, together with combination of a variety of forms, which is harmonious and unified, concise and generous and disclosing the romantic charm of traditional Northern Chinese folk dwellings. The engraving of the architectural elements on the bracket system, Huayazi and sparrowbrace of the wing-room of the courtyard is also quite outstanding (See Figure 6), which adopts the method of combination of deep-relief and bas-relief. Some local positions are also processed with the open work carving and the bracket system part is similar to a polyhedron carving in the form of circular carving, which all enrich changes from different aesthetic perspectives and are allocated with color drawing of flowers, plants and cloud. The part of sparrow brace is mainly engraved in the form of deep-relief, which is engraved with the content of such Chinese characters as "An Ba Xian" and "Xi Shang Mei Shao" as well as the patterns of flowers, plants and cloud, etc. It is lucky that although part of the wing-room has been destroyed, the remaining part of the wing-room is relatively complete, especially the doors and windows, which are engraved with a lot of well-distributed dermatoglyphic patterns. This is also a common form in decoration of doors and windows in folk dwellings in Shaanbei. The pane is matched with engraving of plum blossom or peony on the background of the composition of a straight line or curve, which adds vividness to the doors and windows with the waffle pattern that have rigorous and regular appearance.

From a holistic perspective, the wood carving works maintained in Dangshi manor is not only simple, but also delicate and complicated. After a long history, although there is quite some part of the manor that has been destroyed and missed, it can still reveal the magnificent and exquisite charm that is characteristic of folk dwelling architectural decoration in Shaanbei.

\section{Conclusion}

To sum up, we can see that as one of the typical and traditional folk dwellings in Shaanbei, Dangshi manor employs a lot of techniques in its own engraving skills. Its manifestation content is not only rich and various, but also has subtle changes according to different courtyards. Its perfect grasp on the spatial scale of the engraving elements and the mutual organic connection build a well-proportioned atmosphere that is no loss of compactness. Thus, it becomes a precious inheritance that can not be duplicated in the traditional Chinese folk dwelling culture. Staring from the perspectives of aesthetics, history and architectural decoration, a detailed and sufficient analysis and study of Dangshi manor will have a referential effect on inheritance of traditional architectural decoration art and its application in modern living environment.

\section{References}

Baidu Wikipedia. Stone Carving in Suide. [Online] Available: http://baike.baidu.com/view/1269402.html?wtp=tt Li, M. (2006). Study on Architectural Decoration Art in Folk Dwellings in Shaanbei. Xi'an University of Architecture and Technology, 05: 11. 
Li, Y. J. (2011). Traditional Folk Building and Residential Customs Culture in Shaanbei. Beijing: Science Press, 06: 137.

Wu, W. (2006). Study and Thought on Drum-shaped Bearing Stone in Traditional Residential Gate. Hunan: Furniture \& Interior Design Journal House, 05: 92.

Yue, S. J. (2010). Study on Engraving Art in Folk Dwelling of Shaanxi. Da Jia, 08: 39.

Zhang, Y. C. (2009). Study on Tile Carving Art in Entrance Screen of Traditional Northern Chinese Folk Dwellings and Analysis of Case Study. Xi'an: Xi'an Academy of Fine Arts, 03: 5.

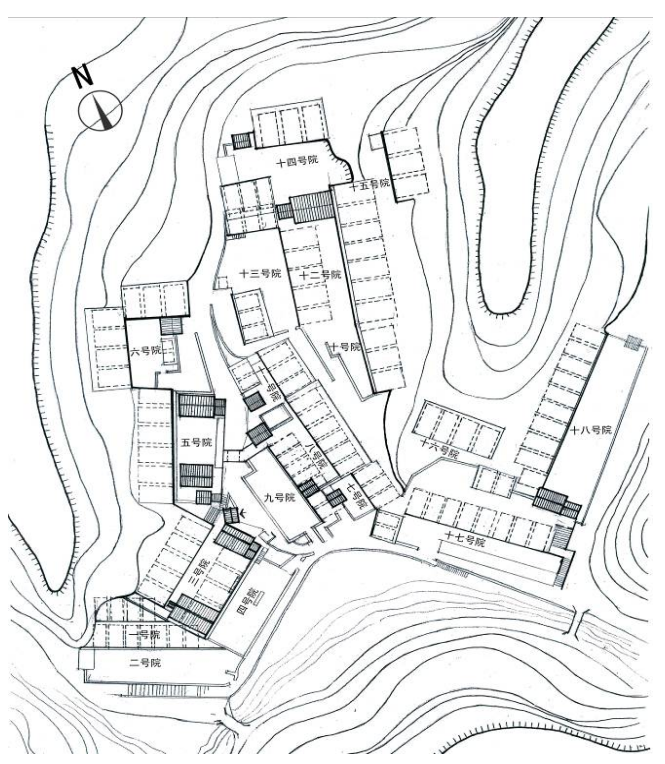

Figure 1. Plane graph of Dangshi manor (source: drawn by the authors)

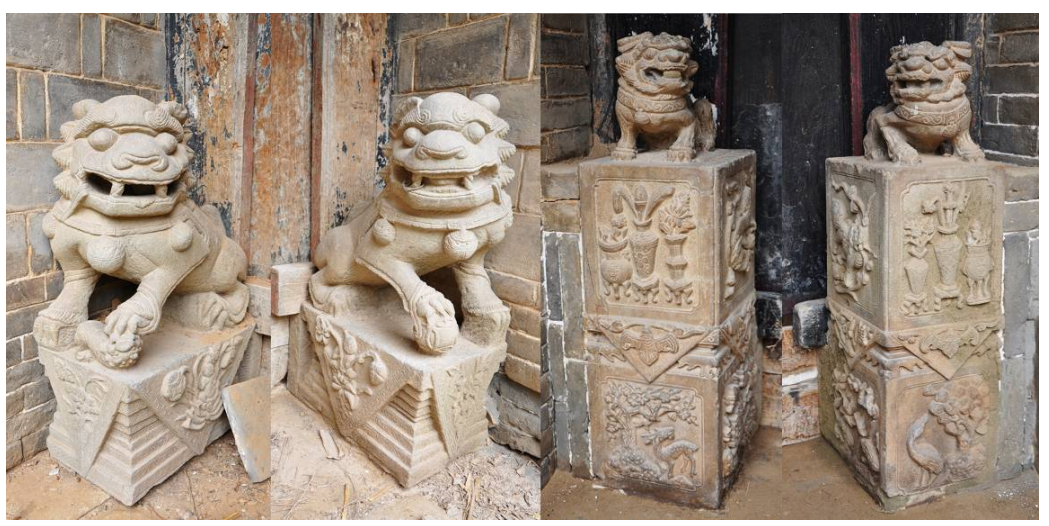

Figure 2. Stone carving lion on the bearing stone (source: drawn by the authors)

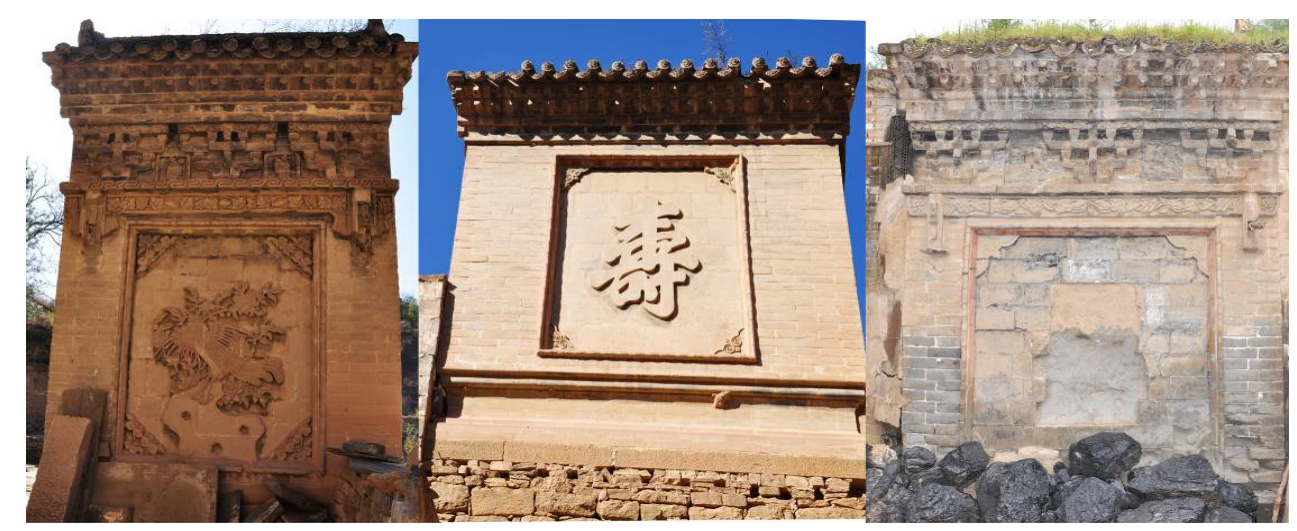

Figure 3. Tile carving of the screen wall in Dangshi manor (source: drawn by the authors) 


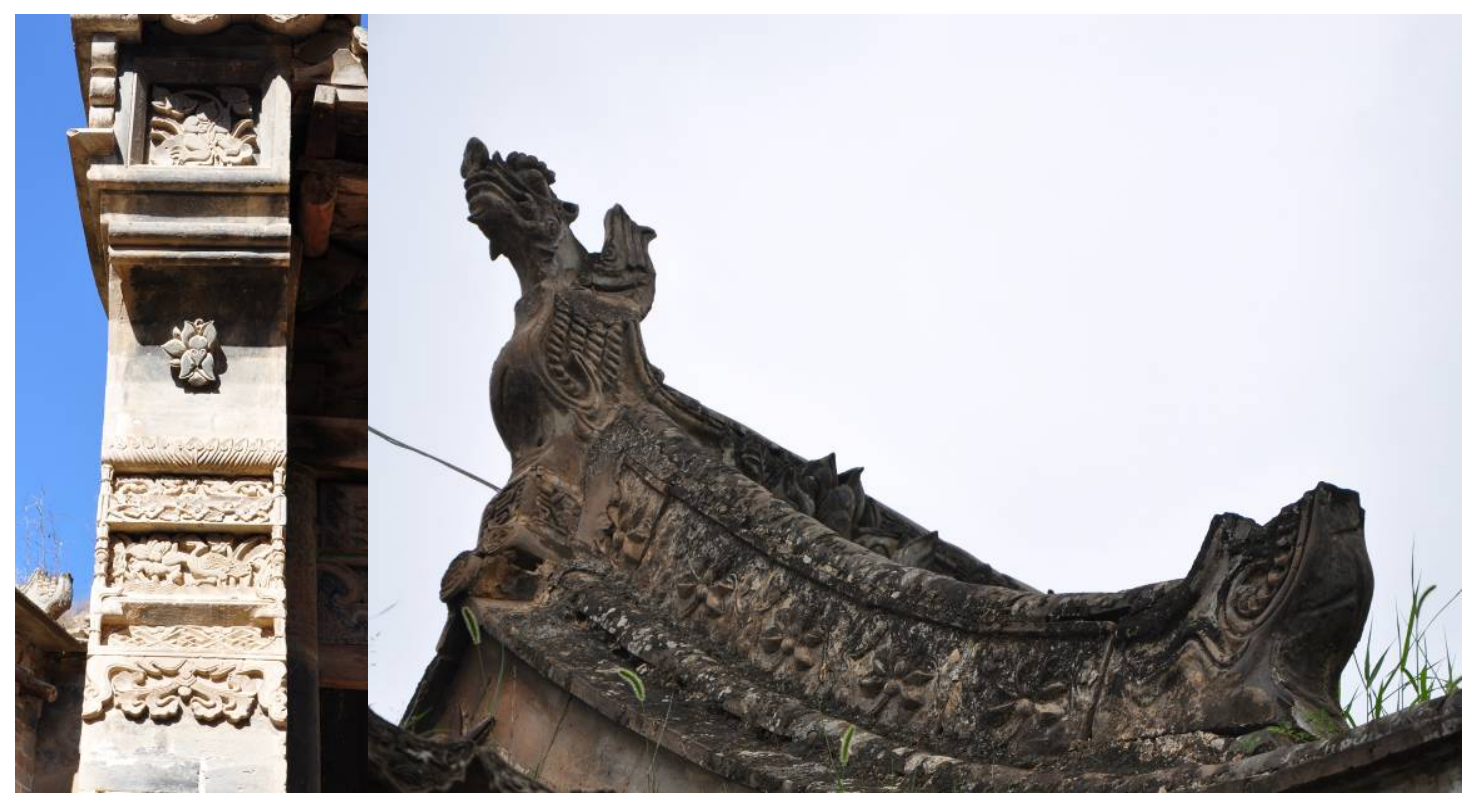

Figure 4. Tile carving of Chitou and ridge (source: drawn by the authors)

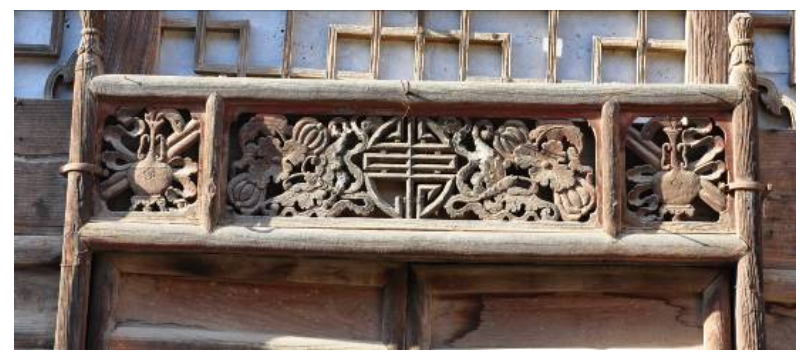

Figure 5. Wood carving of the lintel in the front cave dwelling of the major courtyard (source: drawn by the authors)

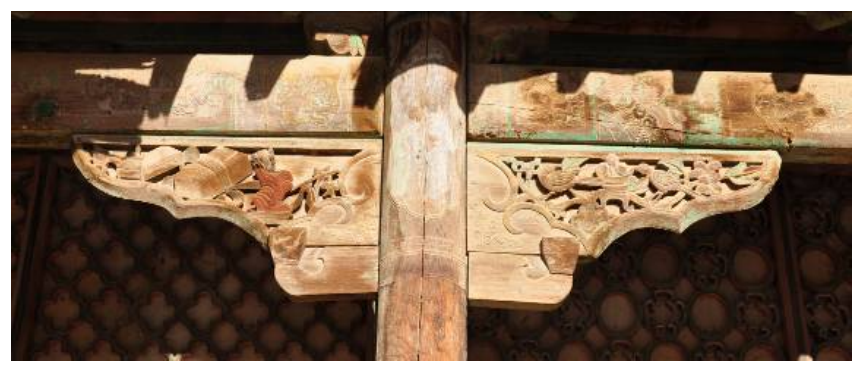

Figure 6. Wood carving of the sparrow brace in the wing-room of the major courtyard (source: drawn by the authors) 